\title{
MCT8 mutation analysis and identification of the first female with Allan-Herndon-Dudley syndrome due to loss of MCT8 expression
}

Suzanna Gerarda Maria Frints ${ }^{* 1,2,12}$, Steffen Lenzner ${ }^{3,12}$, Mareike Bauters ${ }^{4}$, Lars Riff Jensen ${ }^{3}$, Hilde Van Esch ${ }^{5}$, Vincent des Portes ${ }^{6}$, Ute Moog ${ }^{1}$, Merryn Victor Erik Macville ${ }^{1}$, Kees van Roozendaal ${ }^{1}$, Constance Theresia Rimbertha Maria Schrander-Stumpel ${ }^{1,2}$, Andreas Tzschach ${ }^{3}$, Peter Marynen ${ }^{4}$, Jean-Pierre Fryns ${ }^{5}$, Ben Hamel ${ }^{7}$, Hans van Bokhoven ${ }^{7}$, Jamel Chelly $^{8}$, Chérif Beldjord ${ }^{8}$, Gillian Turner ${ }^{9}$, Jozef Gecz $^{10}$, Claude Moraine ${ }^{11}$, Martine Raynaud ${ }^{11}$, Hans Hilger Ropers ${ }^{3}$, Guy Froyen ${ }^{4}$ and Andreas Walter Kuss ${ }^{\star, 3}$

\begin{abstract}
${ }^{1}$ Department of Clinical Genetics, University Hospital azM Maastricht, Maastricht, The Netherlands; ${ }^{2}$ Institute for Growth and Development, GROW, Maastricht University, Maastricht, The Netherlands; ${ }^{3}$ Department of Human Molecular Genetics, Max Planck Institute for Molecular Genetics, Berlin, Germany; ${ }^{4}$ Human Genome Laboratory, Department for Molecular and Developmental Genetics, VIB, Department of Human Genetics, K.U.Leuven, Leuven, Belgium; ${ }^{5}$ Center for Human Genetics, Department of Human Genetics, University Hospital Gasthuisberg, Leuven, Belgium; ${ }^{6}$ Service de Neuropédiatrie, Hospices Civils de Lyon, Lyon, France; ${ }^{7}$ Department of Human Genetics, Radboud University Nijmegen Medical Centre, Nijmegen, The Netherlands; ${ }^{8}$ INSERM Institut Cochin (IC), Département de Génétique et Pathologie Moléculaire GDPM, Equipe de Génétique et Physiopathologie du Retard Mentaux GPRM, Paris, France; ${ }^{9}$ NSW GOLD Service, Hunter Genetics, University of Newcastle, Waratah, New South Wales, Australia; ${ }^{10}$ Department of Genetic Medicine and Departments of Paediatrics and Molecular Biosciences, Women's and Children's Hospital and University of Adelaide, Callahan, South Australia, Australia; ${ }^{11}$ Unité de Génétique, CHU Bretonneau, Tours, France
\end{abstract}

Mutations in the thyroid monocarboxylate transporter 8 gene (MCT8/SLC16A2) have been reported to result in X-linked mental retardation (XLMR) in patients with clinical features of the Allan-HerndonDudley syndrome (AHDS). We performed MCT8 mutation analysis including 13 XLMR families with LOD scores > 2.0, 401 male MR sibships and 47 sporadic male patients with AHDS-like clinical features. One nonsense mutation (c.629insA) and two missense changes (c.1A $>T$ and c.1673G $>A$ ) were identified. Consistent with previous reports on MCT8 missense changes, the patient with c.1673G $>$ A showed elevated serum T3 level. The c.1A $>$ T change in another patient affects a putative translation start codon, but the same change was present in his healthy brother. In addition normal serum T3 levels were present, suggesting that the c.1A $>$ T (NM_006517) variation is not responsible for the MR phenotype but indicates that MCT8 translation likely starts with a methionine at position p.75. Moreover, we characterized a de novo translocation $t(X ; 9)(q 13.2 ; p 24)$ in a female patient with full blown AHDS clinical features including elevated serum T3 levels. The MCT8 gene was disrupted at the X-breakpoint. A complete loss of MCT8

\footnotetext{
${ }^{*}$ Correspondence: Dr SGM Frints, Department of Clinical Genetics, Maastricht University Medical Center UMC ${ }^{+}$, PB 5800, Maastricht 6202 AZ, The Netherlands. Tel: + 3143 3875855; Fax: + 3143 3875800;

E-mail: suzanne.frints@gen.unimaas.nl and

Dr AW Kuss, Department of Human Molecular Genetics, Max Planck Institute for Molecular Genetics, Berlin, Germany. Tel: +49 30 84131253;

Fax: + 4930 84131383;

E-mail: kuss a@molgen.mpg.de

${ }^{12}$ These authors contributed equally to this work.

Received 22 November 2007; revised 22 February 2008; accepted 28 February 2008; published online 9 April 2008
} 
expression was observed in a fibroblast cell-line derived from this patient because of unfavorable nonrandom X-inactivation. Taken together, these data indicate that MCT8 mutations are not common in non-AHDS MR patients yet they support that elevated serum T3 levels can be indicative for AHDS and that AHDS clinical features can be present in female MCT8 mutation carriers whenever there is unfavorable nonrandom X-inactivation.

European Journal of Human Genetics (2008) 16, 1029-1037; doi:10.1038/ejhg.2008.66; published online 9 April 2008

Keywords: MCT8; Allan-Herndon-Dudley syndrome; XLMR; mutation analysis; expression; X-inactivation

\section{Introduction}

The thyroid monocarboxylate transporter 8 (MCT8) gene is located at Xq13.2 and mutations have previously been reported in mentally retarded men with psychomotor retardation ${ }^{1}$ as well as with thyroid and neurological abnormalities. ${ }^{2,3}$ This clinical entity was earlier reported as the Allan-Herndon-Dudley syndrome (AHDS; OMIM 300523) and Schwartz et $a l^{4}{ }^{4}$ Maranduba et $a l^{5}$ as well as Herzovich $^{6}$ described additional patients with MCT8 mutations.

In early childhood the clinical features of male patients with MCT8 mutations include severe developmental delay, truncal hypotonia with poor head control, quadriplegia (cerebral palsy) or spastic paraplegia with paroxysmal dyskinesia or choreoathetosis of arms and hands. Elevated serum 3,3',5-tri-iodothyronine (T3) levels are present, but can remain undetected because serum thyroxine (tetraiodothyronine (T4)) and thyroid-stimulating hormone (TSH) can fall within the normal range. In late childhood, the clinical phenotype is characterized by severe mental retardation (MR), (nearly) no speech, spastic paraplegia with hyperreflexia or clonus, poor head/neck control through increased truncal hypotonia and muscle weakness. $^{7}$ Clinical variability within and between MCT8 mutation families has been reported. ${ }^{8}$ The reported MCT8 mutation female carriers showed normal growth and psychomotor development, they had normal facial appearance, and they did not present with neurological symptoms. This is most likely explained by normal random X-inactivation or favorable nonrandom X-inactivation. ${ }^{7}$

In the previously published patients, MCT8 was screened because of high serum T3 levels detected in these patients, but the exact relation with the different clinical characteristics is not yet understood. Serum (free) T3 levels are not routinely measured in diagnostic laboratories and it is thus not known to which extent loss of function or hypomorphic MCT8 proteins play a role in cognitive disabilities. Schwartz $\mathrm{et}^{4} \mathrm{l}^{4}$ have speculated that MCT8 mutations may rank among the more prevalent X-chromosomal causes of MR. In order to determine whether MCT8 mutations also occur in non-AHDS MR patients we performed MCT8 sequence analysis in a cohort of XLMR families and male
MR sibships ${ }^{9}$ in comparison with sporadic MR patients presenting AHDS(-like) clinical features. In total 461 men with MR were investigated.

Moreover, we report on the first AHDS female patient with loss of MCT8 expression due to the disruption of this gene by a balanced $t(X ; 9)(q 13.2 ; p 24)$ translocation.

\section{Patients and methods \\ Patients}

Informed consent was obtained from the parents of the affected patients ${ }^{9}$ and genomic DNA was isolated from peripheral blood according to standard procedures. DNA was dissolved in TE buffer at a concentration of $0.33 \mu \mathrm{g} / \mu \mathrm{l}$ and stored at $4^{\circ} \mathrm{C}$. For clinical description see results.

\section{MCT8 mutation analysis}

The MCT8 gene structure was defined through analysis of genomic (GenBank AL157934) and cDNA (GenBank NM_006517.2) sequences (http://www.ncbi.nih.gov/), using Vector NTi software (Informax, Bethesda, MD, USA).

For the 401 individuals from affected male MR sibships and the 13 index patients from XLMR families, ${ }^{9}$ the exon and splice site regions were PCR amplified (primer sequences and PCR conditions are available upon request) and submitted to denaturing high-performance liquid chromatography (DHPLC) analysis as previously described. ${ }^{10}$ Sequencing reactions were then carried out for patient DNAs, which showed abnormal elution profiles in the DHPLC analysis.

For the 47 sporadic MR patients, mutation screening of MCT8 was performed by direct sequence analysis of all six coding exons with their flanking intronic sequences at positions $>100 \mathrm{bp}$ away from the splice sites. The PCR primers and conditions (available upon request) used here were obtained from Dr E Friesema (exon 1) and Professor Dr C Schwartz (exons 2-6). Sequencing reactions were performed with either of both PCR primers using the BigDye v3.1 sequencing kit on an ABI PRISM $3130 \times 1$ capillary sequencer (Applied Biosystems). Nucleotide changes were verified by sequencing the opposite strand from a new PCR product. 
Breakpoint analysis in patient with de novo balanced 46,X,t(X;9)(q13.2;p24)

Analysis of the Xq-breakpoint in the female patient was performed by standard fluorescent in situ hybridization (FISH) on metaphase chromosome spreads of the patient. Genomic BAC clones, obtained from the Sanger Centre (http://www.sanger.ac.uk/Teams/Team63/), were labeled with the BioNick Translation kit (Invitrogen) with Texas red. Clone RP11-100N10, which maps at 9p21.1, was labeled with FITC (green) and used as a reference probe. Signals were visualized by digital imaging microscopy with Cytovision capturing software (Applied Imaging, Santa Clara, CA, USA).

\section{$\mathrm{X}$-inactivation studies}

Lymphocyte genomic DNA of the female subjects was subjected to the androgen-receptor gene methylation assay for assessment of the methylation status, as described by Allen et al. ${ }^{11}$ After adding a ROX-labeled genotyping marker 100-500 (Applied Biosystems), the samples were separated on an ABI PRISM 3100 automated DNA sequencer (Applied Biosystems) and analyzed with the GeneScan analysis software (Applied Biosystems) for peak position and area intensity calculations. These data were further processed in Excel.

Late replication studies were performed on chromosomes in (pro)metaphase, through $5^{\prime}$-bromodeoxyuridine (BrdU) incorporation. ${ }^{12}$ Subsequently, the inactive, latereplicating $\mathrm{X}$ chromosome was visualized by an anti-BrdU Alexa 488-antibody (Molecular Probes) combined with an anti-digoxigenin-rhodamine $\mathrm{Fab}$ fragment antibody (Roche) against the X-centromere XbamH5-dig (modified protocol after Garcia-Heras et $a l^{12}$ and Coonen et $a l^{13}$ ). Chromosome 9 was painted in red using a commercial probe, WCP9-Spectrum Orange (Vysis).

\section{Real-time quantification of MCT8 mRNA levels}

Total RNA from $10^{7}$ skin fibroblasts was extracted with $1 \mathrm{ml}$ TRIzol (Invitrogen) according to the manufacturer's instructions. Genomic DNA was removed by DNaseI (Roche) treatment for $30 \mathrm{~min}$ at room temperature. Total RNA $(1 \mu \mathrm{g})$ was reverse transcribed with Superscript II (Invitrogen) in a final volume of $20 \mu \mathrm{l}$. Real-time quantification was performed in $25 \mu \mathrm{l}$ reactions on $1 \mu \mathrm{l}$ cDNA product with the $2 \times$ qPCR MasterMix Plus for SYBR Green I dNTP (Eurogentec, Belgium), and the MCT8 primer set (MCT8 forward: 5'-GCTGCCCTTGGCTACTTTGT-3' and MCT8 reverse: 5'-TCTGAGAACTCCTCCTCCACATACT-3' which span exons 3 and 4 ), or the $\beta$-actin primer set for normalization ( $\beta$-actin forward: 5'-CACCCTGAAGTACCC CATCG- $3^{\prime}$ and $\beta$-actin reverse: $5^{\prime}$-TGCCAGATTTTCTC CATGTCG-3', which are both located within exon 3 ). The final concentration of each primer was $500 \mathrm{nM}$. After an initial denaturation step of $10 \mathrm{~min}$ at $95^{\circ} \mathrm{C}$, thermal cycling conditions were $15 \mathrm{~s}$ at $95^{\circ} \mathrm{C}$ and $1 \mathrm{~min}$ at $60^{\circ} \mathrm{C}$ for 40 cycles. Finally, the dissociation curves for each reaction were determined. All samples were run in duplicate on an ABI PRISM 7000 instrument (Applied Biosystems) and two independent runs were performed for all samples. For data analysis, the threshold cycle $\left(C_{t}\right)$ values were exported to Excel and processed according to the comparative $\mathrm{ddCt}$ method using the $\beta$-actin $C_{\mathrm{t}}$ values for normalization (Sequence Detection System bulletin no. 2; Applied Biosystems).

\section{Results \\ Patients}

A total of 461 male MR patients were investigated, 13 being from XLMR families with linkage intervals (LOD > 2.0) overlapping the MCT8 locus, 401 from male MR sibships including 2-5 affected first degree male relatives and 47 sporadic MR patients presenting AHDS(-like) clinical features collected by the University Hospital of Leuven, Belgium. The including criteria for the selection of AHDS (-like) clinical features were severe MR, absence of speech, axial hypotonia and spasticity. As controls we used 240 unrelated healthy men.

Of the 13 families with linkage data, 6 have been published earlier: MRX26, ${ }^{14}$ MRX31, ${ }^{15}$ MRX65, ${ }^{16}$ T003, T040 and T050. ${ }^{17}$ The remaining seven XLMR families as well as the 401 male MR sibships were obtained through the EuroMRX Consortium (http://www.euromrx.com/); see also de Brouwer et al. ${ }^{9}$ Specific clinical information for the families where putatively causative changes in MCT8 were identified in this study is given below.

Family TOO3 All affected men (Figure 1a; II:1; III:8; IV:1; IV:2) in family T003 suffered from early onset encephalopathy with severe developmental delay, hypotonia, choreoathetosis of arms and hands, spasticity and they were bedridden. In early adulthood, male III:8 had an asthenic habitus with a flat chest and developed scoliosis (secondary short stature). All affected men died of unknown cause at age 5 months (IV:2), 3 years 3 months (II:1), 5 years (IV:1) and 22 years (III:8). No T3 levels could be determined, as all affected family members including all obligate female carriers were deceased at the time of this study. All possible female carriers showed normal random X-inactivation. ${ }^{17}$

Family P030 The index patient (Figure 1b; II:2) of family P030 was born preterm at 35 weeks of gestation with birth weight $2500 \mathrm{~g}$. He had transient breathing distress. He suffered from an early onset encephalopathy (before 4 months of age) and clinical investigation at age 8 years showed cachexia, axial hypotonia, spastic quadriplegia (bedridden) and he had no speech. He died at the age of 14 years of ileus. Thyroid hormone testing was not done in this patient. His younger brother (Figure 1b; II:3) suffered 


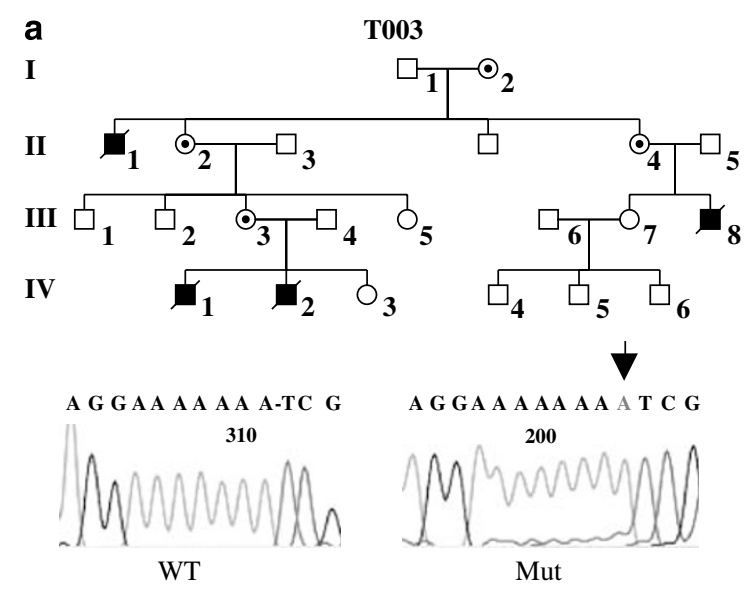

b

P030

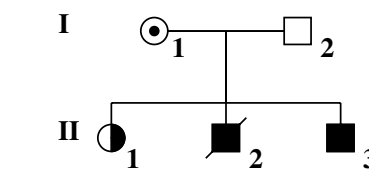

C T T T G C C G GT GT GC C

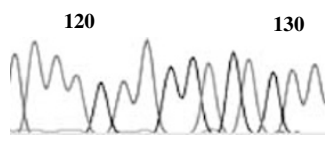

WT

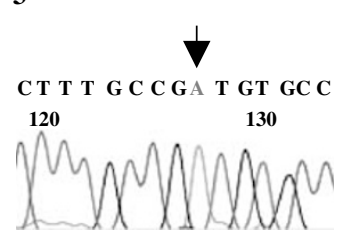

Mut

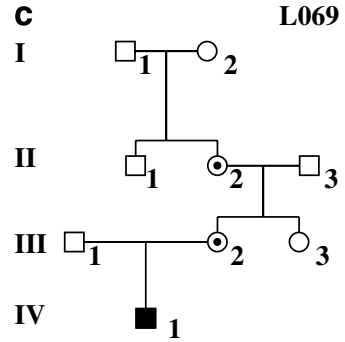

GA T G ACA T G G G G A

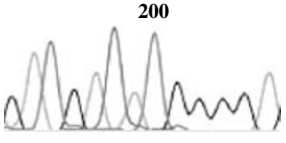

WT

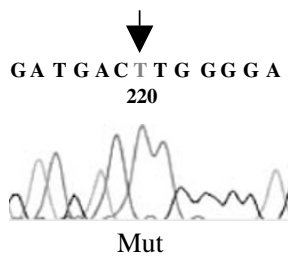

Mut

Figure 1 Pedigrees of families with MCT8 mutations. Family T003 (a), Family P30 (b) and Family L069 (c). For each family the pedigree and chromatograms of the sequence section containing the observed change are shown. 'WT' denotes the wild-type sequence. 'Mut' marks the patient derived sequence containing the changed base, which is highlighted in red and indicated by an arrow.

from severe neonatal encephalopathy with spastic quadriplegia, axial hypotonia and choreoathetotic upper limb movements. At the age of 13 years, he was severely mentally retarded, did not have any speech and he was not able to walk. His eye contact was good. He had elevated free T3 levels, low free T4 and normal TSH (Table 1). His sister (Figure 1b; II:1) suffered from mild MR without neurological clinical features. Thyroid hormone tests revealed elevated serum T3, low free T4 and normal TSH (Table 1). The mother (I:1) was mentally normal. Apart from a slight decrease of free $\mathrm{T} 4$ and elevated thyroxine binding globulin, other thyroid hormone concentrations were normal (Table 1). These women were not available for $\mathrm{X}$-inactivation studies.

A female patient with de novo balanced $46, \mathrm{X}, \mathrm{t}(\mathrm{X} ; 9)$ (q13.2;p24) translocation was severely developmentally retarded. She had axial hypotonia and developed athetoid movements of the upper limbs and spastic paraplegia of the legs. Later in life, facial dysmorphism became more evident with hypotonia, elongation and open mouth with prominent teeth (Figure 2). She had nearly no speech. She suffered from scoliosis and contractures of knees and ankles. She showed elevated serum and free T3. Serum T4 was normal, free T4 was slightly elevated and TSH level was very low (Table 1). For more clinical details see supplementary text (only electronically available).

\section{MCT8 mutation screening in 13 XLMR families who map at the MCT8 locus}

Mutation analysis of the six exons and their adjacent intronic sequences of MCT8 in 13 XLMR families with linkage intervals overlapping the MCT8 gene, showed an insertion of an A nucleotide (c.629insA) in family T003 (Figure 1a), which causes a frame shift in exon 1 and introduces a premature termination codon in exon 2 (p.K210fsX241).

\section{MCT8 mutation screening in 401 male MR sibships}

In two of the 401 families with 2-5 affected first degree male relatives, we detected nucleotide changes that were 
not present in the control panel: In family P030 a missense change c.1673G $>$ A, p.Gly558Asp was detected (Figure 1b) which segregated with MR in the family. In family L069 a missense change affecting the putative translational start codon (c.1A>T). The c.1A > T change in family L069 was however, also present in a healthy brother of the patient (Figure 1c). In addition, we identified a silent variation (c.669C > T, p.Leu223Leu) in family A019, which occurred only once in the patient panel and was not present in controls or in dbSNP.

\section{MCT8 mutation screening in 47 sporadic male patients with AHDS(-like) clinical features}

In one sporadic patient we identified a nucleotide change in intron 2 (c. 964-35G $>$ A), which was not a known SNP. An additional synonymous sequence variant (c.1818C $>$ T, p.Ser606Ser) that was also not registered in dbSNP was found in several MR patients and controls.

\section{Breakpoint analysis in female with AHDS-like phenotype}

In a female patient with AHDS clinical features, cytogenetic analysis revealed a de novo balanced translocation: 46,X,t(X;9)(q13.2;p24). FISH analysis with BAC clone RP11-644H1, containing the proximal $100 \mathrm{~kb}$ (exons 1 and 2) of the MCT8 gene, showed a split signal at the Xq-breakpoint (Figure 3). Late-replication cytogenetic studies revealed that the derivative $\mathrm{X}$ chromosome was $100 \%$ active in cultured lymphocytes (Figure 4 ). The same result was obtained by standard androgen receptor gene assay, which yielded a ratio of 100/0 between the derivative and the normal $\mathrm{X}$ chromosome, respectively (data not shown).

\section{MCT8 expression analysis in female with balanced $\mathrm{t}(\mathrm{X} ; 9)(\mathrm{q} 13.2 ; \mathrm{p} 24)$}

Real-time quantitative PCR analysis of MCT8 expression analysis using primers spanning exons 3 and 4, with normalization towards $\beta$-actin mRNA levels, demonstrated high levels of MCT8 in control skin fibroblasts $\left(C_{\mathrm{t}}=22\right)$, while the expression was low in blood leukocytes, platelets, or hair roots $\left(C_{\mathrm{t}}>32\right.$, data not shown). We analyzed MCT8 expression levels in the fibroblasts of the female patient with $\mathrm{t}(\mathrm{X} ; 9)(\mathrm{q} 13.2 ; \mathrm{p} 24)$ and discovered a 250 -fold reduction of expression $\left(C_{\mathrm{t}}=30\right)$ as compared to gender-matched controls (they were not age matched; Figure 5). These data are in agreement with a complete nonrandom inactivation of the normal X chromosome resulting in the loss of MCT8 expression in this female AHDS patient.

\section{Discussion}

To date, about 20 families with MCT8 gene mutations have been described. ${ }^{7}$ The three additional changes we describe in this study include one nonsense mutation (c.629insA) 

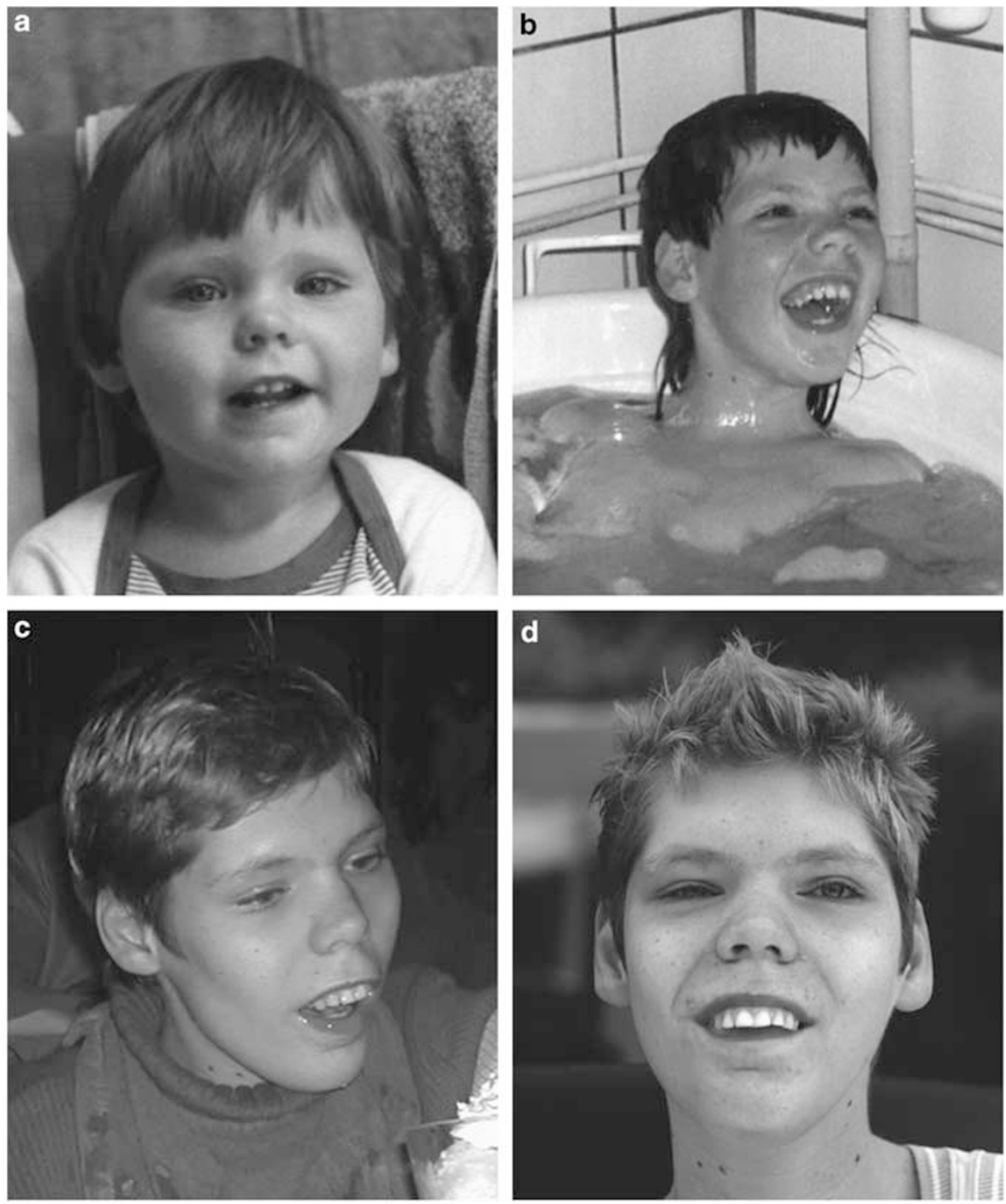

Figure 2 Facial appearance of a female with Alan-Herndon-Dudley syndrome (AHDS) and de novo translocation t(X;9)(q13.2;p24) at 20 months (a), 5 years (b), 17 years (c) and 27 years (d) of age, showing the changing phenotype. Her face elongated gradually and myopathy developed. She has long palpebral fissures, midfacial hypoplasia, a depressed broad nasal bridge, anteverted nares with bulbous nasal tip and an open large mouth (for further clinical details see supplementary text).

and two missense changes (c.1A $>\mathrm{T}$ and c.1673G $>\mathrm{A}$ ). Considering that only the two missense changes were found in 401 male MR sibships without any linkage information and none in the 47 sporadic MR male patients our results suggest that, contrary to earlier assumptions, MCT8 mutations are not a common cause for XLMR.

Clinical AHDS characteristics often include axial hypotonia, severe MR without speech/developmental delay, spastic paraplegia but a large clinical inter- and intra-familial variability exists. Due to the limited number of families with MCT8 mutations, an extensive genotype-phenotype correlation study has not been performed yet. However, based on the available literature, individual clinical features do not seem to correlate with specific MCT8 mutations except for elevated levels of serum T3, which are the most consistent feature in AHDS male patients and it has previously been put forward to use the assessment of T3 as a method for screening a high-risk MR population. ${ }^{4}$ We therefore determined serum T3 in the two patients with missense changes in MCT8 but only found an increased concentration in the patient carrying the c.1673G $>$ A change, which segregated with MR in the family. An in silico estimation of its impact on the three-dimensional protein structure and function using the Polyphen tool (http://genetics.bwh.harvard.edu/pph/; Sunyaev et al ${ }^{18}$ ) predicts this change to be possibly damaging. 

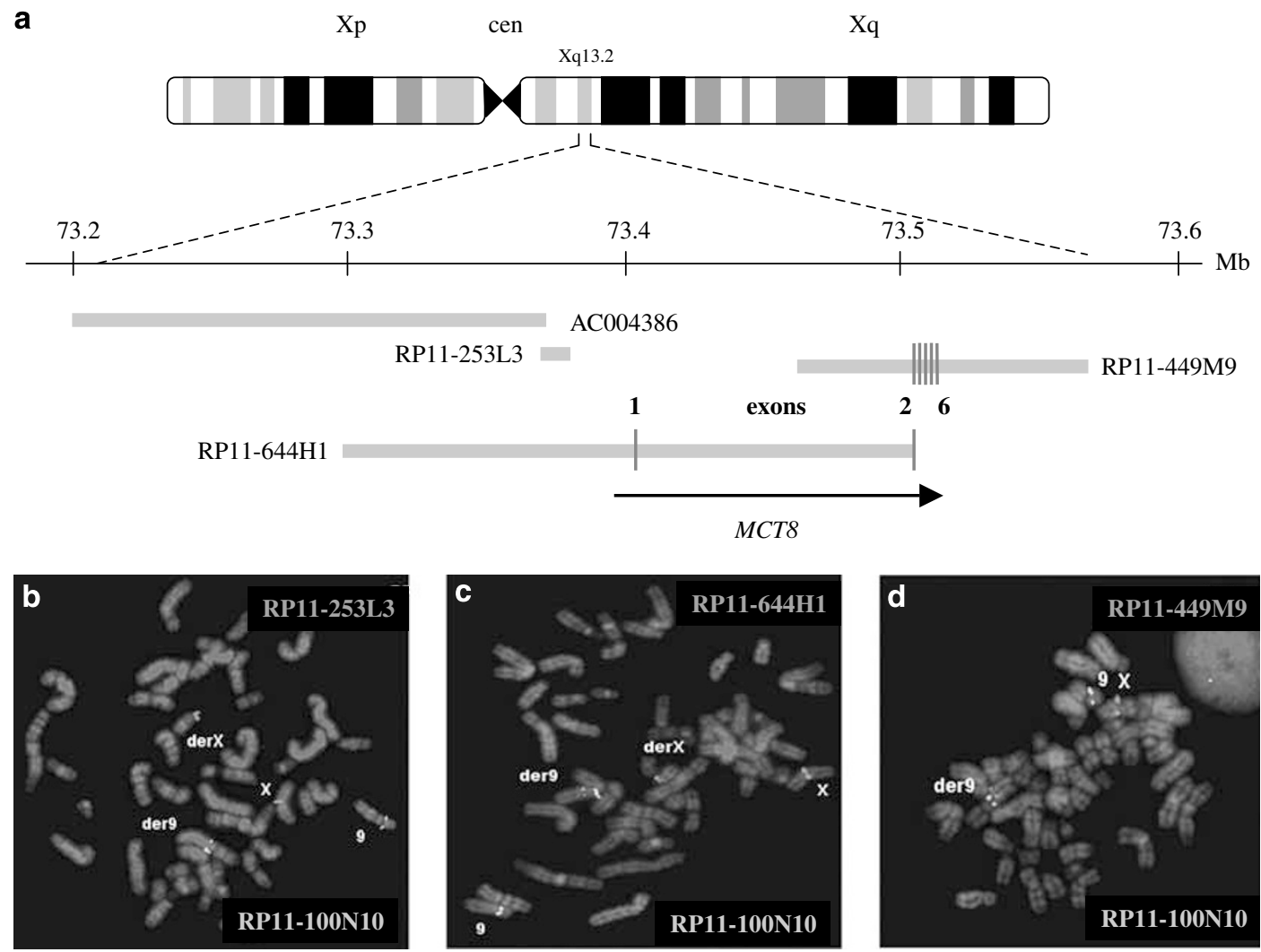

Figure 3 Analysis of the $X q$-breakpoint in the patient with $t(X ; 9)(q 13.2 ; p 24)$. Schematic representation of the Xq13.2 genomic region with the position on $\mathrm{X}$ indicated in $\mathrm{Mb}(\mathbf{a})$. The position of the genomic clones as well as the exons of the MCT8 gene within these clones is indicated. FISH data with the Xq13.2-derived BAC clones (red signals) RP11-253L3 (b), RP11-644H1 (c) and RP11-449M9 (d) on metaphase chromosomes of the patient. Clone RP11-100N10, which maps at 9p21.1, was used as a reference probe (green signals).

The c.1A>T (NM_006517) change found in the patient from family L069 does not cosegregate with the disease, and as the T3 levels in this patient are normal (Table 1) it is unlikely that the change accounts for the observed MR phenotype. This finding is nevertheless noteworthy, as it implies that the methionine considered to mark the translation start in NM_006517 19,20 is likely not the relevant translation start codon and therefore provides evidence sustaining the earlier suggestion that another methionine at position p.75 might be the start codon for the MCT8 protein.

The synonymous sequence variant (c.669C $>$ T) we discovered in family A019 most likely does not affect the gene product. Fibroblast cell-lines were not available for MCT8 expression investigation. The findings are in agreement with the observation that apart from MR, there are no additional clinical features indicating that the patient is suffering from AHDS.

We also investigated a de novo balanced $\mathrm{t}(\mathrm{X} ; 9)(\mathrm{q} 13.2 ; \mathrm{p} 24)$ translocation in a female patient with elevated serum T3 levels. FISH-based mapping showed that her X-breakpoint is located in MCT8 intron 1. Expression studies in fibroblasts demonstrated complete loss of MCT8 expression in this patient. Therefore, the situation in this female reflects a male status with a MCT8 nonsense mutation. This is the first woman reported with an obvious AHDS phenotype. About 25\% (4 of the 16 tested females) of the MCT8 mutation female carriers show thyroid functional problems with elevated serum T3 levels. ${ }^{7}$ Six affected MCT8 mutation female carriers have been reported: one female carrier described in this study (P030, Figure 1b; II.2) with mild to severe MR, family Fi with mild MR in maternal grandmother and severe MR in maternal aunt, ${ }^{2,21}$ one affected female carrier in family K9248 with unexplained prenatal injury ${ }^{4}$ and two carrier females; a mother with mild developmental delay and her daughter with severe MR, hydrocephalus and myelomeningocele. ${ }^{6}$ We believe that unfavorable nonrandom $\mathrm{X}$-inactivation can occur in MCT8 mutation female carriers leading to AHDS clinical features and that MR can be part of the phenotype in MCT8 mutation carriers. However, most MCT8 mutation female carriers show random $\mathrm{X}$-inactivation resulting in normal thyroid and cognitive function. ${ }^{7}$ 


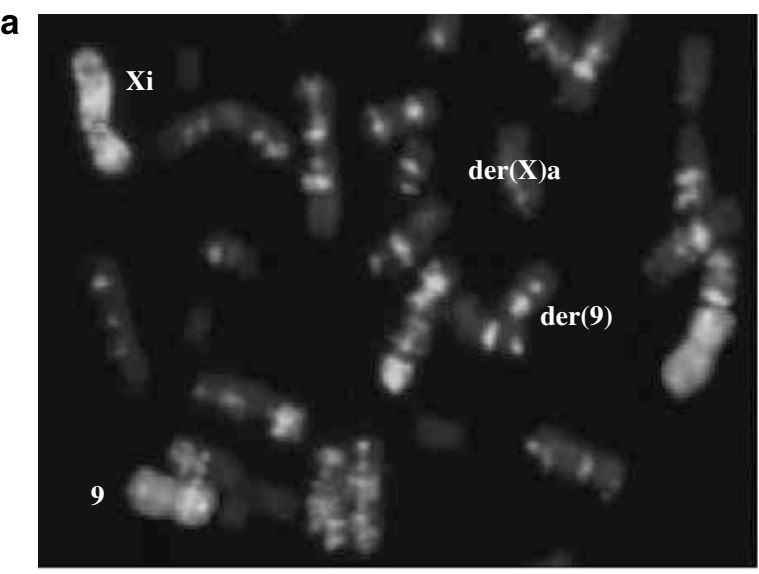

b

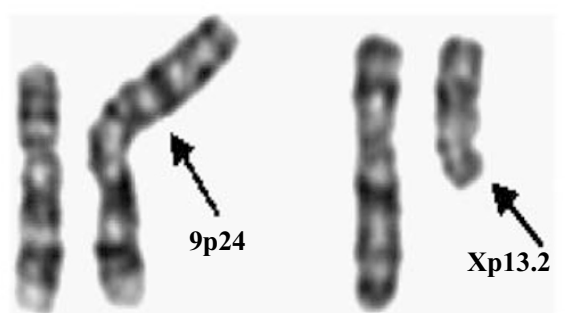

Figure 4 Late replication study in female with de novo balanced $46, X, t(X ; 9)(q 13.2 ; p 24) . X i$ is inactivated $X$ chromosome and $X a$ is active $X$ chromosome. With FISH analysis combined with immunocytochemistry ( $X$ centromere probe in red, incorporated BrdU in green) all blood lymphocytes investigated showed $100 \%$ inactivation of the normal $\mathrm{X}$ chromosome because this is the late replication $\mathrm{X}$ chromosome with most BrdU incorporated (a). Der(9) is the aberrant chromosome 9. In the lower part of the picture a partial GTG banded karyotype is presented (b).

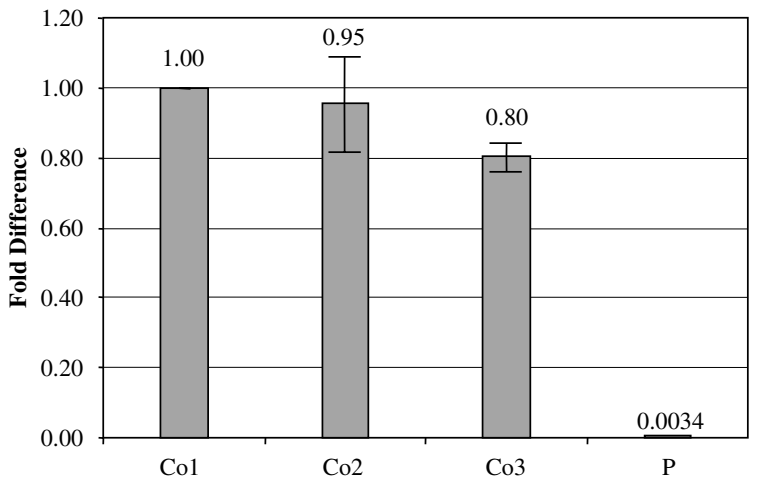

Figure 5 MCT8 expression levels in fibroblasts. Expression of MCT8 mRNA, by real-time quantification, in skin fibroblasts of the present female patient $(P)$ relative to that of two male and one female controls (Co1, Co2 and Co3, respectively). The fold differences are given in comparison with $\mathrm{Co} 1$ and are the mean of two independent experiments. The cycle at which the threshold was reached $\left(C_{t}\right.$ value) with the MCT8 primer pair was 22 for the controls and 30 for the patient.

Taken together, our findings indicate that MCT8 mutations are not a frequent cause of nonsyndromic XLMR. In addition, they suggest that serum T3 levels should be determined in patients with AHDS(-like) phenotype before screening MCT8 for mutations. In females, AHDS can occur in a situation where there is a MCT8 gene defect in combination with elevated T3 level and unfavorable nonrandom X-inactivation.

\section{Acknowledgements}

We thank Mieke Alofs (University Hospital Maastricht) and Marleen Willems (University Hospital Leuven) for their expert assistance on culturing fibroblasts, Erik van Lierop, Bertien Hollanders, Lucy Amory and Demis Tserpelis (University Hospital Maastricht) for cytogenetic and molecular $X$-inactivation studies. We are grateful to Bettina Moser, Melanie Wendehack and Marianne Schlicht for technical assistance in the mutation screening study. This work was supported by the Research Grant 016.066.017 of the Dutch NWO/ZonMW VENI fund, The Netherlands, Research Grant G-0229-01 of the Fund for Scientific Research-Flanders (FWO-Vlaanderen), Belgium, by the Deutsche Forschungsgemeinschaft (SFB 577) and the EURO-MRX project (part of the European 'Quality of Life and Management of Living Resources Programme' QLG3-CT-2002-01810).

\section{References}

1 Friesema EC, Grueters A, Biebermann H et al: Association between mutations in a thyroid hormone transporter and severe X-linked psychomotor retardation. Lancet 2004; 364: 1435-1437.

2 Brockmann K, Dumitrescu AM, Best TT, Hanefeld F, Refetoff S: $\mathrm{X}$-linked paroxysmal dyskinesia and severe global retardation caused by defective MCT8 gene. J Neurol 2005; 252: 663-666.

3 Holden KR, Zuniga OF, May MM et al: X-linked MCT8 gene mutations: characterization of the pediatric neurologic phenotype. J Child Neurol 2005; 20: 852-857.

4 Schwartz CE, May MM, Carpenter NJ et al: Allan-HerndonDudley syndrome and the monocarboxylate transporter 8 (MCT8) gene. Am J Hum Genet 2005; 77: 41-53.

5 Maranduba CM, Friesema EC, Kok F et al: Decreased cellular uptake and metabolism in Allan-Herndon-Dudley syndrome (AHDS) due to a novel mutation in the MCT8 thyroid hormone transporter. J Med Genet 2006; 43: 457-460.

6 Herzovich V, Vaiani E, Marino R et al: Unexpected peripheral markers of thyroid function in a patient with a novel mutation of the MCT8 thyroid hormone transporter gene. Horm Res 2007; 67: $1-6$.

7 Schwartz CE, Stevenson RE: The MCT8 thyroid hormone transporter and Allan-Herndon-Dudley syndrome. Best Pract Res Clin Endocrinol Metab 2007; 21: 307-321.

8 Friesema EC, Jansen J, Heuer H, Trajkovic M, Bauer K, Visser TJ: Mechanisms of disease: psychomotor retardation and high T3 levels caused by mutations in monocarboxylate transporter 8 . Nat Clin Pract Endocrinol Metab 2006; 2: 512-523.

9 de Brouwer AP, Yntema HG, Kleefstra $\mathrm{T}$ et al: Mutation frequencies of X-linked mental retardation genes in families from the EuroMRX consortium. Hum Mutat 2007; 28: 207-208.

10 Jensen LR, Lenzner S, Moser B et al: X-linked mental retardation: a comprehensive molecular screen of 47 candidate genes from a 7.4 Mb interval in Xp11. Eur J Hum Genet 2007; 15: 68-75.

11 Allen RC, Zoghbi HY, Moseley AB, Rosenblatt HM, Belmont JW: Methylation of HpaII and HhaI sites near the polymorphic CAG repeat in the human androgen-receptor gene correlates with $\mathrm{X}$ chromosome inactivation. Am J Hum Genet 1992; 51: 1229-1239.

12 Garcia-Heras J, Martin JA, Witchel SF, Scacheri P: De novo $\operatorname{der}(\mathrm{X}) \mathrm{t}(\mathrm{X} ; 10)(\mathrm{q} 26 ; \mathrm{q} 21)$ with features of distal trisomy 10q: case report of paternal origin identified by late replication with BrdU 
and the human androgen receptor assay (HAR). J Med Genet 1997; 34: $242-245$.

13 Coonen E, Dumoulin JC, Dreesen JC, Bras M, Evers JL, Geraedts JP: Clinical application of FISH for sex determination of embryos in preimplantation diagnosis of X-linked diseases. J Assist Reprod Genet 1996; 13: 133-136.

14 Robledo R, Melis P, Laficara F et al: Further linkage evidence for localization of mutational sites for nonsyndromic types of $\mathrm{X}$-linked mental retardation at the pericentromeric region. $\mathrm{Am} \mathrm{J}$ Med Genet 1996; 64: 107-112.

15 Donnelly AJ, Partington MW, Ryan AK, Mulley JC: Regional localisation of two non-specific X-linked mental retardation genes (MRX30 and MRX31). Am J Med Genet 1996; 64: 113-120.

16 Yntema HG, van den Halm B, Knoers NV et al: X-linked mental retardation: evidence for a recent mutation in a five-generation family (MRX65) linked to the pericentromeric region. Am J Med Genet 1999; 85: 305-308.
17 Raynaud M, Moizard MP, Dessay B et al: Systematic analysis of X-inactivation in 19 XLMR families: extremely skewed profiles in carriers in three families. Eur J Hum Genet 2000; 8: 253-258.

18 Sunyaev S, Ramensky V, Koch I, Lathe III W, Kondrashov AS, Bork P: Prediction of deleterious human alleles. Hum Mol Genet 2001; 10: $591-597$.

19 Friesema EC, Ganguly S, Abdalla A, Manning Fox JE, Halestrap AP, Visser TJ: Identification of monocarboxylate transporter 8 as a specific thyroid hormone transporter. J Biol Chem 2003; 278: 40128-40135.

20 Lafreniere RG, Carrel L, Willard HF: A novel transmembrane transporter encoded by the XPCT gene in Xq13.2. Hum Mol Genet 1994; 3: 1133-1139.

21 Dumitrescu AM, Liao XH, Best TB, Brockmann K, Refetoff S: A novel syndrome combining thyroid and neurological abnormalities is associated with mutations in a monocarboxylate transporter gene. Am J Hum Genet 2004; 74: 168-175.

Supplementary Information accompanies the paper on European Journal of Human Genetics website (http://www.nature.com/ejhg) 